\title{
Milli-arcsecond images of the Herbig Ae star HD 163296
}

\author{
S. Renard ${ }^{1}$, F. Malbet ${ }^{1}$, M. Benisty ${ }^{2}$, E. Thiébaut ${ }^{3}$, and J.-P. Berger ${ }^{4,1}$ \\ ${ }^{1}$ Laboratoire d'Astrophysique de Grenoble, CNRS-UJF UMR5571, BP 53, 38041 Grenoble, France \\ e-mail: [Stephanie.Renard;Fabien.Malbet]@obs.ujf-grenoble.fr \\ 2 INAF - Osservatorio Astrofisico di Arcetri, Largo E. Fermi 5, 50125 Firenze, Italy \\ e-mail: benisty@arcetri.astro.it \\ 3 Centre de Recherche Astrophysique de Lyon, CNRS-UCBL-ENSL UMR5574, 69561 St-Genis-Laval, France \\ e-mail: thiebaut@obs.univ-lyon1.fr \\ 4 European Southern Observatory, Alonso de Cordova, 3107, Vitacura, Chile \\ e-mail: jpberger@eso.org
}

Received 30 April 2010 / Accepted 15 June 2010

\begin{abstract}
Context. The very close environments of young stars are the hosts of fundamental physical processes, such as planet formation, stardisk interactions, mass accretion, and ejection. The complex morphological structure of these environments has been confirmed by the now quite rich data sets obtained for a few objects by near-infrared long-baseline interferometry.

Aims. We gathered numerous interferometric measurements for the young star HD 163296 with various interferometers (VLTI, IOTA, KeckI and CHARA), allowing for the first time an image independent of any a priori model to be reconstructed.

Methods. Using the Multi-aperture image Reconstruction Algorithm (MiRA), we reconstruct images of HD 163296 in the $H$ and $K$ bands. We compare these images with reconstructed images obtained from simulated data using a physical model of the environment of HD 163296.

Results. We obtain model-independent $H$ and $K$-band images of the surroundings of HD 163296. The images detect several significant features that we can relate to an inclined asymmetric flared disk around HD 163296 with the strongest intensity at about 4-5 mas. Because of the incomplete spatial frequency coverage, we cannot state whether each of them individually is peculiar in any way. Conclusions. For the first time, milli-arcsecond images of the environment of a young star are produced. These images confirm that the morphology of the close environment of young stars is more complex than the simple models used in the literature so far.
\end{abstract}

Key words. instrumentation: interferometers - techniques: image processing - stars: pre-main sequence stars: individual: HD 163296

\section{Introduction}

The process of star formation triggered by the collapse and fragmentation of a molecular cloud leads to the birth of a young star surrounded by a circumstellar disk and outflows. The disks are believed to be the place where the planets form (Boss 1997; Mayer et al. 2002), and are composed of a mixture of gas and dust with a wide range of grain composition (Henning \& Meeus 2009). The standard picture of the close environment of premain sequence stars is so far limited to a quasi-stationary accreting disk partially reprocessing the irradiation from the central protostar with potential planetary gaps opened by newly formed planets. Some of the accreted material is ejected by means of bipolar outflows but their precise origin has not yet been identified. Most of the models are presently assumed to be symmetric around the star rotation axis and stationary. However hydrodynamical turbulence, gravitational waves, magneto-rotational instabilities, vortices and thermal instabilities occurring on the AU-scale are known to play a major role in star and planet formation (e.g., Balbus \& Hawley 1991). The close environments of young stars are therefore not expected to be as simple as they are currently modeled, but the observational measurements do not provide tight enough constraints to unambiguously identify strong departures from axisymmetrical models.
The photometric and spectroscopic observations obtained with very modest spatial resolution are usually integrated over a subarcsecond field of view which corresponds to several tens of AUs at the distance of the closest star formation regions $(140 \mathrm{pc})$. Observations at the scale of $1 \mathrm{AU}$ and below correspond to 7 milli-arcsec (mas) angular scale and requires therefore optical long-baseline interferometry. However, even if long-baseline optical interferometry is capable of reaching very high angular resolution, the observations are usually limited to a small number of measurements (see Millan-Gabet et al. 2007, for a review). With the advent of interferometers with more than two telescopes and with significantly high spectral resolution (e.g., VLTI/AMBER, CHARA/MIRC), the number of interferometric observations has significantly increased allowing the first images to be reconstructed with aperture synthesis techniques. Images of stellar surfaces (e.g., Monnier et al. 2007; Haubois et al. 2009; Zhao et al. 2009), binaries (e.g., Zhao et al. 2008; Kraus et al. 2009), or circumstellar shells around evolved stars (e.g., Le Bouquin et al. 2009) have been obtained mostly for objects brighter than the brightest young stars. In the young stellar object field, we are at a comparable stage to that reached 40 years ago when the first 3 antennas of the VLA became operational producing the first radio-interferometry images (Hogg et al. 1969). We report the first attempt to reconstruct an image of a circumstellar disk 
around a young star using mostly AMBER/VLTI interferometric data.

In this study, we present reconstructed images of the young star HD 163296 (MWC 275), an isolated Herbig Ae star (HAe) of spectral type A1, with a $\sim 30 L_{\odot}$ luminosity, and a $\sim 2.3 M_{\odot}$ mass located at $122_{-13}^{+17}$ pc (van den Ancker et al. 1998; Natta et al. 2004; Montesinos et al. 2009). In scattered light (Grady et al. 2000) and at millimeter wavelengths (Mannings \& Sargent 1997), a disk has been detected on large scales, traced out to 540 AU. The CO millimeter line observations have revealed a large-scale inclined disk in Keplerian rotation probably evolving towards a debris disk phase (Isella et al. 2007). HD 163296 also exhibits an asymmetric outflow perpendicular to the disk, with a chain of six Herbig-Haro knots (HH409) tracing the history of mass loss (Devine et al. 2000; Wassell et al. 2006). The emission of the innermost regions, observed in far-UV emission lines, have been attributed to optically thin gas accreting onto the stellar surface, a magnetically confined wind, or shocks at the base of the jet (Deleuil et al. 2005; Swartz et al. 2005). HD 163296 has been observed with several interferometers (see Benisty et al. 2010, B10 hereafter). Using the largest set of interferometric data of a young star, we present here the first reconstructed images of a complex young stellar object.

The article is organized as follow. Sect. 2 describes the image reconstruction method and the methodology employed to extract the best reconstructed image. Section 3 presents the reconstructed images obtained in the $H$ and $K$ bands and their analysis using simulated data generated from inner disk models. In Sect. 4, we describe the choices made during the image reconstruction process, and discuss the physical meaning of the features seen in the image, as well as the consequences for the models commonly used. Finally, Sect. 5 summarizes our results and provides some perspectives for the future.

\section{Image reconstruction}

\subsection{Image reconstruction by MiRA}

The principle of interferometry is to interfere coherently the light coming from a single astronomical source from two or more independent telescopes (Lawson 2000; Malbet \& Perrin 2007). An interferometer measures a complex number referred to as the visibility. According to the Van Cittert-Zernicke theorem, this complex visibility, $V \mathrm{e}^{\mathrm{i} \phi}$, is the Fourier transform of the object brightness distribution at the spatial frequency of the observations, given by the projected baseline in units of wavelength $(B / \lambda)$. The visibility amplitude, $V$, is related to the spatial extent of the emission, while the phase, $\phi$, provides the location of the photocenter. However, the requested infrastructure to carry out optical interferometry is complex and has led to a limitation in the number of telescopes (Baldwin \& Haniff 2002). The main consequence is to provide a sparse sampling of the spatial frequencies, the socalled $(u, v)$ plane. Moreover, the absolute value of the phase $\phi$ is lost due to atmospheric turbulence that randomly modifies it. However, by adding the phases of the fringes measured for each baseline over a 3-telescope configuration, one can measure an additional quantity, the closure phase, which is insensitive to the atmospheric disturbance (Monnier 2003; Monnier et al. 2006). The closure phase includes part of the Fourier phase information, and is related to the global asymmetry of the emission: a point-symmetric object has a zero closure phase. The main observables are therefore the squared visibility amplitudes, $V^{2}$, and the closure phases $(\mathrm{CP})$.
The objective of the image reconstruction is to numerically retrieve an approximation of the true brightness distribution of the source given the interferometric measurements. To account for the data, the Fourier transform of the sought image should fit the measured complex visibilities. However, due to the sparse $(u, v)$ coverage, the image reconstruction problem is ill-posed as there are more unknowns, e.g., the pixels of the image, than measurements. Additional prior constraints are therefore required to supplement the available data and retrieve a unique and stable solution. A very general solution is to define the optimal image to be the solution to the optimization problem (Thiébaut 2005; Thiébaut \& Giovannelli 2009):

$$
\min _{\boldsymbol{x}}\left\{f_{\text {data }}(\boldsymbol{x})+\mu f_{\text {prior }}(\boldsymbol{x})\right\} \quad \text { with } \quad \boldsymbol{x} \geqslant 0 \text { and } \sum_{\mathrm{n}} \boldsymbol{x}_{\mathrm{n}}=1
$$

where $\boldsymbol{x} \in \mathbb{R}^{N}$ are the pixel values in the discretized image and $N$ the number of pixels. In problem (1) the strict constraints $x \geqslant 0$ and $\sum_{n} \boldsymbol{x}_{n}=1$ account for the non-negativity and the normalization of the brightness distribution. The objective function in the optimization problem (1) is a joint criterion of two components:

- Minimization of the likelihood term $f_{\text {data }}(\boldsymbol{x})$, which enforces agreement of the sought image with the data. In practice, this term is derived from the noise statistics. For instance, it is the $\chi^{2}$ of the data for Gaussian statistics.

- Minimization of the regularization term $f_{\text {prior }}(\boldsymbol{x})$, which favors images that are the simplest or the smoothest according to a priori assumptions.

The parameter $\mu>0$ is used to tune the relative importance of the two terms so as to select the most regular image among all those compatible with the data.

Many different algorithms have been developed to solve the image reconstruction problem from optical interferometry data (e.g., Cotton et al. 2008; Thiébaut \& Giovannelli 2009). In this paper, we use the Multi-Aperture Image Reconstruction Algorithm (MiRA; by Thiébaut 2008). MiRA is capable of dealing with any available interferometric data (complex visibilities, squared visibilities, closure phases, etc.), and has been successfully used to process real data (e.g., Lacour et al. 2008; Lacour et al. 2009; Le Bouquin et al. 2009; Haubois et al. 2009). When phases are missed because of the atmospheric turbulence, the MiRA algorithm can directly fit the available interferometric observables without explicitly rebuilding the missing phases. Finally, one can choose the most effective of various regularization methods integrated into MiRA for the type of object observed and check the effect of the regularization on the resulting image. MiRA directly attempts to solve the problem in Eq. (1) using an iterative non-linear optimization algorithm (Thiébaut 2002). Because of the missing phases, the function to be minimized is not however convex thus has several local minima. The final image is therefore determined by the data, the choice of the regularization (and its level), and the initial image.

\subsection{Methodology used in this work}

Systematic tests have been performed on the MiRA algorithm by Renard et al. (in prep.; RTM10 hereafter) in which images of ten astrophysical objects were reconstructed, for different $(u, v)$ coverages and signal-to-noise ratios. Twelve regularizations were tested and images were reconstructed for a set of weight factors $\mu$. The optimal solution is the one that minimizes 

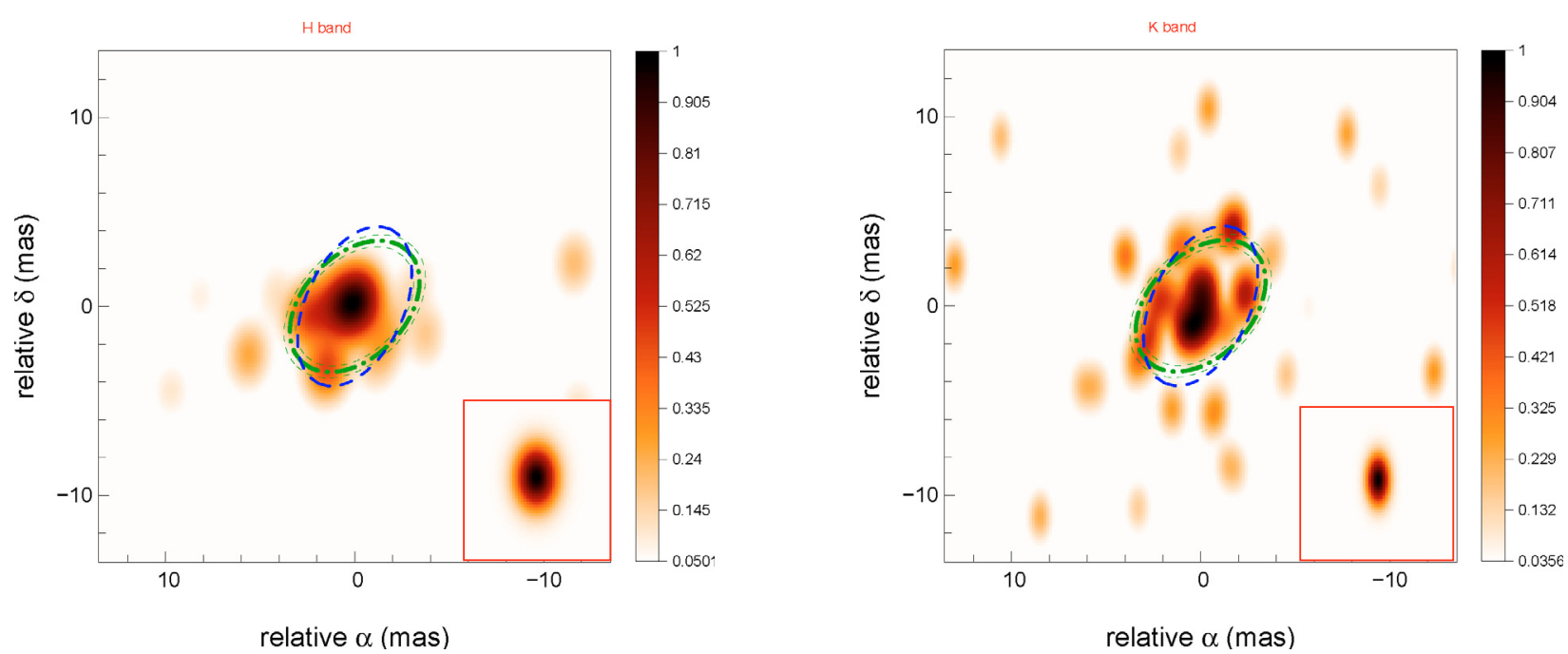

Fig. 1. Reconstructed images of HD 163296 in the $H$ (left) and $K$ bands (right), after a convolution with a Gaussian beam at the interferometer resolution. The colors are scaled to the squared root of the intensity with a cut corresponding to the maximum expected dynamic range (see text for details). The blue ellipse traces the location of the main secondary blobs, and the green dot-dashed ellipse corresponds to the location of the rim in the B10 model, with its width given by the green dashed ellipses. North is up and east is left. The sub-panel in the right corner of each plot indicates the Gaussian beam at the interferometer resolution, applicable to Figs. 3, 4, and 6.

the mean-squared distance between the model and the reconstructed image. The RTM10 tests led to the following conclusions:

- The total variation regularization (Strong \& Chan 2003), which minimizes the total norm of the image gradient, is the best regularization method in most of the cases.

- The weight factor $\mu$ depends, within one order of magnitude, on neither the amount of data, the signal-to-noise ratio, nor the object type, but only on the type of the regularization used. Each regularization has its best value for $\mu$.

The chosen regularization for this work is therefore the total variation and the weight factor $\mu$ is set to be $10^{2}$. The effect of changing $\mu$ is discussed in Appendix B. As explained above, because part of the phase information is lost, the image reconstruction from squared visibilities and closure phases yields to a criterion with multiple local minima. The solution depends on the initial image used to start the iterative process. Different starting images, such as a Dirac or a random flux distribution among pixels, were tested and, thanks to a good regularization term and a sufficient number of data points, the solutions obtained were always found to be the same. Here, we choose a symmetric Gaussian as a starting image.

Once the parameters of the image reconstruction process are set, the images can be reconstructed. The solutions are not straightforward to analyze because of artifacts caused by the image reconstruction process or the quality of the data set, e.g., voids in the $(u, v)$ plane, error bars. There are no objective criteria to distinguish between the actual structures from the object and the artifacts caused only by the data structure. We therefore performed a comparative analysis between our results and the results obtained from simulated data from the B10 model for HD 163296. To do so, we simulated fake data sets, using the B10 model and the same $(u, v)$ plane and errors as in the real data set. Image reconstruction was also performed for the simulated data in the same conditions and compared to the image model. This comparative method is important to understand what could be trusted in the actual reconstructed images and what could not.

\subsection{Interferometry data set}

The principal characteristics of the data set is summarized in this section. A more detailed description is given in the Appendix A. HD 163296 was observed with several interferometers. The large data set comprises $H$ and $K$-band data from VLTI (Benisty et al. 2010), IOTA (Monnier et al. 2006), Keck-I (Monnier et al. 2005), and CHARA (Tannirkulam et al. 2008). This data set represents the largest set of interferometric data available so far for a young stellar object. Since a trade-off has to be found between enough data to pave the $(u, v)$ plane and the wavelength dependency of the observed object, we decided to reconstruct two different images, one in $H$ and one in $K$ band, using all the spectral channels available in each band i.e. assuming the object to be grey in each band (see Sect. 4.2 for further discussion).

\section{Results and first image analysis}

\subsection{Reconstructed images in the $H$ and $K$ bands}

Using the methodology described in Sect. 2.2, we reconstruct the images of HD 163296 in the $H$ and $K$ bands. To produce a rendu similar to that usually used in radio-interferometry, we convolve all the resulting images with a Gaussian beam at the interferometer resolution defined by the $(u, v)$ plane. The resulting images are plotted in Fig. 1 with a color scale and in Fig. 2 with linear contours. As the minimum cut, we use the level corresponding to the expected dynamic range (see Sect. 4.1 for the discussion on how to compute this value).

At first look, the spot representing the star is unambiguous and corresponds to the maximum of the images in the two bands. Around this central spot, one can see many secondary blobs. In the next section, we will discuss the level of confidence in these blobs by comparing them to the results obtained on simulated data from the B10 model. The main secondary blobs are concentrated in the center of the image around the brightest spot at a distance of smaller than 4-5 mas. To show the location of these secondary blobs, an ellipse is drawn using a dashed blue line in Figs. 1 and 2 with a semi-major axis of 4.5 mas, an inclination of $\sim 55^{\circ}$, and a position angle of $\sim 155^{\circ}$. When considering their 

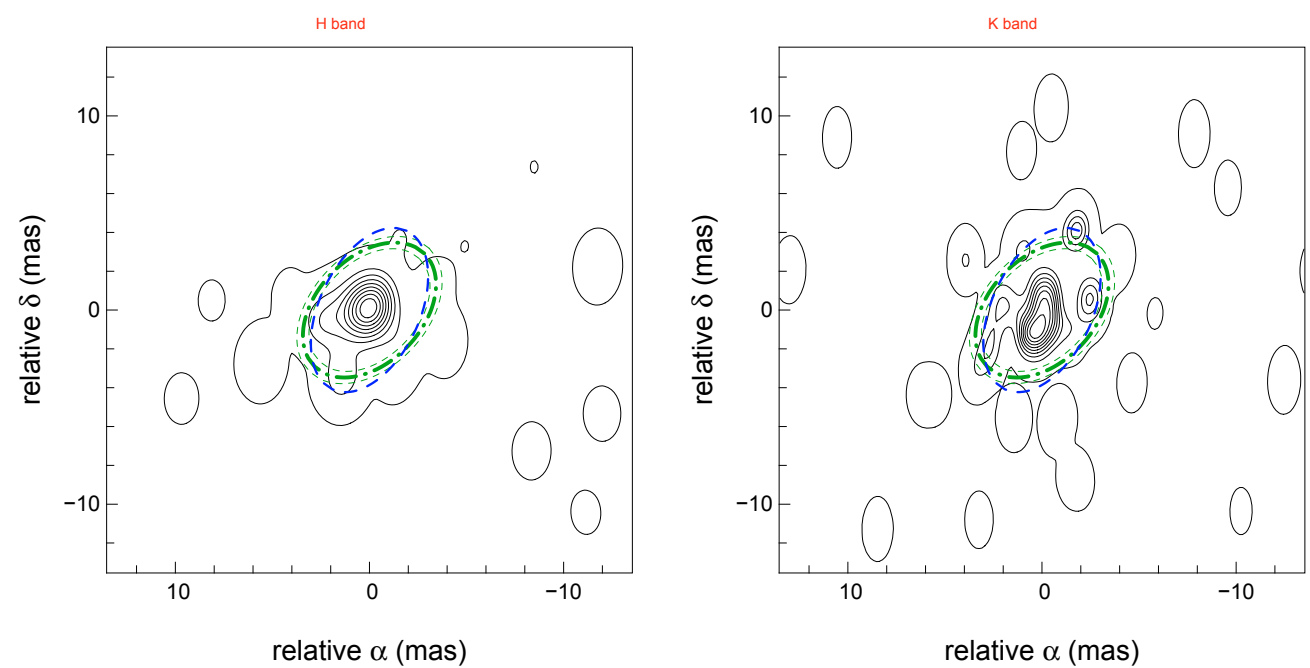

Fig. 2. Contours of the reconstructed images of HD 163296 in the $H$ (left) and $K$ bands (right), after a convolution with a Gaussian beam at the interferometer resolution. The contours vary linearly between the minimum cut corresponding to the maximum expected dynamic range and the image maximum, with a step around 0.1 .
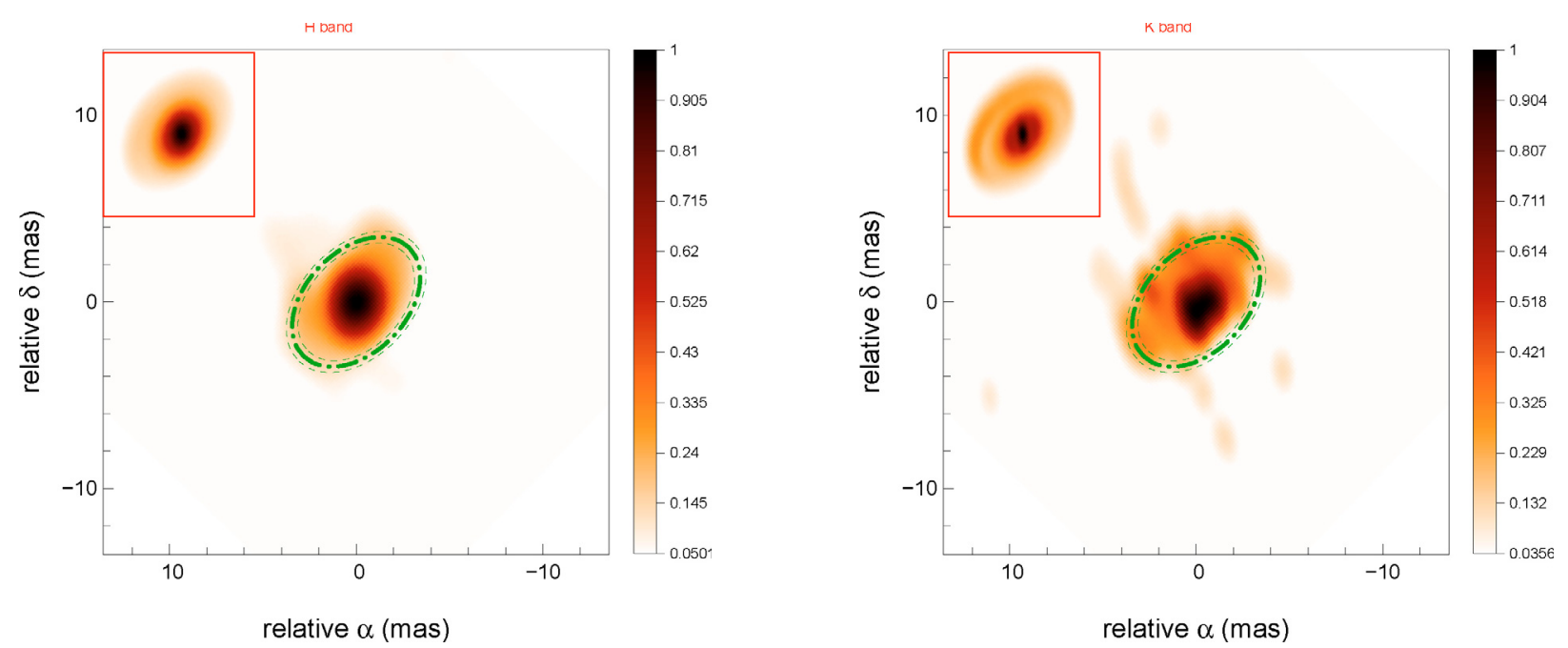

Fig. 3. Reconstructed images of the B10 model of HD 163296 in the $H$ (left) and $K$ bands (right). The dashed green ellipse corresponds to the location of the rim in this model. The models used are presented in the upper left corner. Same conventions as in Fig. 1.

repartition along the ellipse, we find that they are less numerous at the bottom of the ellipse. The percentage of flux in these blobs is around $30 \%$ in the $K$ band and $24 \%$ in the $H$ band.

Inside the ellipse, the central spot is not point-like. In the $H$ band, the central spot is extended, while in the $K$ band the energy is spread into two close separated spots. The emission does not decrease slowly from the central spot towards the exterior but instead shows a rapid decay before increasing again when crossing the ellipse to finally decrease at large distances. We also note that the ellipse and the central spots are not exactly centered, although this result may not be relevant.

\subsection{Comparison with simulated images from models}

To analyze the artifacts in the images, we use the model of HD 163296 presented in B10 (see the bottom right squares of Fig. 3 for the $H$ and $K$-band models) to simulate images with the same conditions as the actual ones. The model is composed of a star (producing $30 \%$ and $14 \%$ of the flux in the $H$ and $K$ bands, respectively, estimated from spectral energy distribution fitting), a dust rim located at $0.45 \mathrm{AU}(\sim 3.6$ mas) representing $16 \%(36 \%)$ in the $H(K)$ band and a bright inner disk, from 0.1 to $0.45 \mathrm{AU}$, contributes to the remaining emission. The reconstructed images of the model from simulated data with the same $(u, v)$ coverage and the same error bars as the real data are shown in Fig. 3 for the $H$ and $K$ bands.

The analysis of the reconstructed images of the model in the $K$ band illustrates that the following structures are well retrieved by the image reconstruction process:

- The dust rim, which is clearly visible at the right location and appears as a somewhat blobby ellipse. We checked that by changing the $(u, v)$ plane filling, the blob location changes but remains aligned along the ellipse.

- The energy inside the disk, which is more spread than if there was only the star inside the dust rim in the model (see below). This emission between the star and the rim represents the bright inner disk.

- The skewness of the dust rim is visible in the reconstructed images: the bottom part of the blobby ellipse has less flux than the top part. 

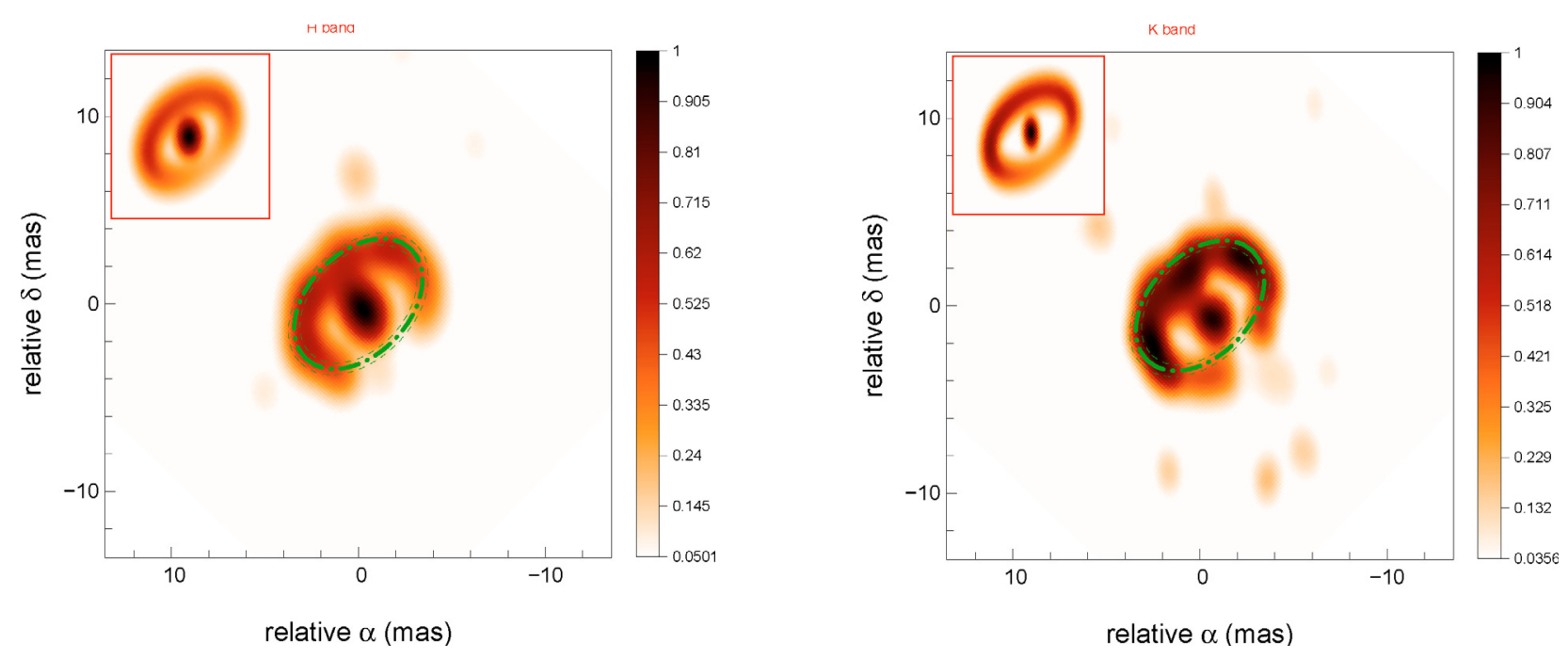

Fig. 4. Reconstructed images of a geometrical model of HD 163296 with only a star plus a Gaussian ring in the $H(l e f t)$ and $K$ bands (right). The dashed green ellipse corresponds to the location of the rim in this model. The models are presented in the upper left corner. Same conventions as in Fig. 1.

- The star, the dust rim, and the inner disk provide $\sim 15 \%$, $\sim 30 \%$, and $\sim 55 \%$ of the flux, respectively. We emphasize that these values are in close agreement with the model.

The only structure that is not retrieved in the image is the hole between the star and the bright inner disk, inside $0.1 \mathrm{AU}$, which is too small to be resolved by the interferometer.

In the $H$ band, the dust rim disappears in the reconstructed image as a ring of blobs, but still seems to define the outer boundary of the object. The main reasons are that the rim represents only $16 \%$ of the flux to be compared with $36 \%$ in the $K$ band, and, that the angular resolution is not high enough in the $H$ band data compared to the $K$ band data (with the CHARA very long baselines). For the dust rim to be seen in the $H$ band, we would need data on longer baselines and higher dynamics in the reconstructed image (1000 at least, 2000 to be unambiguously seen). As in the $K$ band, the bright inner disk is also present in the $H$ band image as a large spot in the middle, which would not have existed with the star only (see below), and represents $86 \%$ of the total flux.

To demonstrate that the bright inner disk is clearly seen in the reconstructed images, i.e., that the central spot includes more energy than that from the star alone, reconstruction of a simpler model is performed. This model is the same as the B10 model but without the bright inner disk, i.e. a star surrounded by a Gaussian ring. The star fluxes in the $H$ and $K$ bands remain the same and the Gaussian ring accounts for $70 \%$ of the flux in the $H$ band and $86 \%$ in the $K$ band. Figure 4 clearly indicates that the star alone does not spread across more than over 4 pixels in the $K$ band and 7 in the $H$ band, which is less than in Fig. 3.

This analysis performed on existing models allows us to state which features in the reconstructed images from Fig. 1 can be trusted. We argue that the main secondary blobs present around the main central spot are real. Their spatial distribution along an ellipse and the intensity present between these peaks and the central spot are also real. However, the clumpy structure of the ring is probably not representative of the reality, but only of the actual $(u, v)$ plane. More observations at different spatial frequencies will probably change the actual position of these peaks along the ellipse, which may be smoothed. However, we conclude that the inclination and orientation of the observed distribution of peaks along an ellipse are real. This orientation and inclination are indeed very close to the ones fitted by B10 and Tannirkulam et al. (2008), and are consistent with previous estimates at different wavelengths (Isella et al. 2007). The second feature that we think is representative of the reconstructed image is that the central spot is extended and not reduced to an unresolved point as a point-like star would be. The shape of this central spot is certainly dependent on the filling of the $(u, v)$ plane, although the position of the centroid is certainly representative of reality.

\section{Discussion}

In this section, we discuss the reconstructed images.

\subsection{Dynamic range}

To compute the theoretical dynamical range of our image, we use an estimator based on the one proposed by Baldwin \& Haniff (2002)

$\sqrt{\frac{n}{(\delta V / V)^{2}+(\delta \mathrm{CP})^{2}}}$,

where $n$ is the total number of measurements, $\delta V / V$ is the relative error in the visibilities, and $\delta \mathrm{CP}$ is the error in the closure phases (in radian). This number indicates the maximum contrast that can be reached in the image given the data, i.e. the ratio of the maximum of the image to the minimum value that can be trusted.

Applying Eq. (2) to the data, a dynamic range of 780 is found in the $K$ band. In the $H$ band, for which there is fewer data and the error bars are slightly larger, a dynamic range of 400 is computed. Minimum cut levels of $1 / 780$ and $1 / 400$, respectively, are applied to all the $K$ and $H$ figures.

\subsection{Use of the spectral information}

The decision to present one reconstructed image in the $H$ and one in the $K$ band results from different tests made on the B10 model. A trade-off has to be made between ensuring that we have enough data to pave the $(u, v)$ plane and the wavelength dependency expected from circumstellar disks. Because of the intrinsic chromaticity of the object, we prefer to reconstruct two separated images in the $H$ and $K$ bands, otherwise two separated 


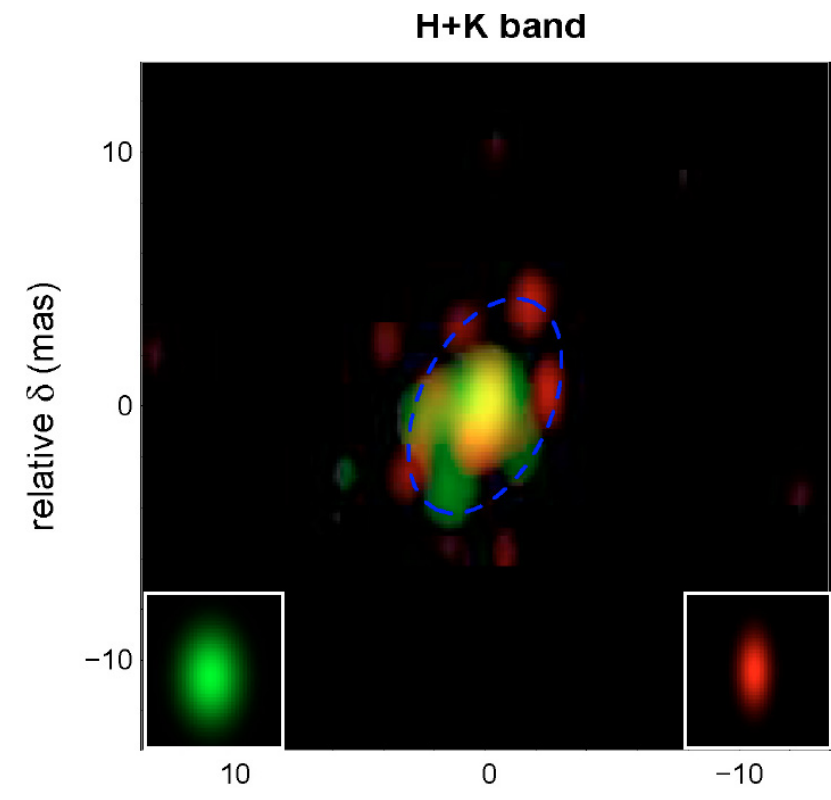

relative $\alpha$ (mas)

Fig. 5. Combination of the reconstructed images of HD 163296 in a two-color image ( $H$ band in green, $K$ band in red). The blue ellipse traces the location of the main secondary blobs of the $K$-band emission. The sub-panels indicate the Gaussian beam at the interferometer resolution used in the convolution.

visibilities, sampling different emitting regions, may correspond to the same spatial frequency. We show in Fig. 5 the combination of both reconstructed images that illustrate complementary features.

For each band, we used all the data points in the different spectral channels, assuming implicitly the object to be grey in each separated band. Compared to the model, this method produces images with fewer artifacts than when reconstructing a single broadband image with the average data for all spectral channels. The $(u, v)$ plane is indeed far more filled taking into account all the wavelengths, because there are more spatial frequencies.

\subsection{Physical consequences for the models}

We emphasize that we have obtained a new type of data in the form of images reconstructed with limited assumptions. The analysis of the results described in Sect. 3.2 is useful for distinguishing artifacts caused mainly by the shape of the $(u, v)$ plane from what we infer to be true features. In this section, we highlight the new information provided by these images, but are also aware that these new pieces of evidence have to be handled with great care.

The ellipse described by the successive blobs in the reconstructed image at a distance of 4-5 mas from the center of the image and underlined by a blue dot-dashed line in Figs. 1 and 2 certainly traces an external ring. The characteristics of this ring are not exactly the same as the rim found in the B10 model plotted with a green dashed line in Figs. 1 and 2. The radius of the ring is $0.55 \mathrm{AU}$ instead of $0.45 \pm 0.05 \mathrm{AU}$ in the $\mathrm{B} 10$ model, the inclination is $55^{\circ}$ instead of $48^{\circ} \pm 2^{\circ}$, and the position angle is $155^{\circ}$ instead of $136^{\circ} \pm 2^{\circ}$. The intensity is close to that of the model with $30 \%$ of the total flux instead of $36 \%$. The location of the blobs differs a little bit between the $H$ and $K$ bands: those in the $H$-band image are closer to the center of the image than those in the $K$-band image. This behavior could be explained by a temperature gradient in the disk, which has a tendency to move the peak of intensity closer to the center for the shorter wavelengths. We probably also need data on longer baselines (equivalent to the $K$-band CHARA data) to resolve unambiguously the external ring in the $H$-band image. This bright ring is not clear evidence of a physical rim, which was proposed by Dullemond et al. (2001). We propose that this feature instead traces an enhancement of the intensity caused by a change in the opacity in the disk probably due to sublimation of dust.

We assume that the number of blobs along this ellipse is representative of the azimuthal distribution of the intensity. Following B10 who in their analysis proposed a model of a rim with a skewed distribution of intensity along the ring (see Fig. 4), we assume that the distribution of light along the blue ellipse indicates that the actual ring of light is less luminous in the south-west direction than in the north-east direction at least in the $K$-band image. In the $H$-band image, there are blobs around the central spot that do not appear in the reconstructed image of the model. Does this mean that there is more flux in the external ring than expected? Is the skewness factor more important because the blobs appeared at one side only? Does the presence of blobs in the south part of the ellipse rather than in the north in the $K$-band image have some significance? To remain on solid ground, we assume that the intensity varies along the distribution of blobs, but do not have definitive data to determine the magnitude of this effect. The departure from the axisymmetry may be caused by an inclined surface of the disk or even a strong dust opacity change.

The energy detected within the ellipse is certainly real: as explained in Sect. 3.2, the central region, which contains about $70 \%$ of the flux, certainly does not originate in a single unresolved star but from an extended source. These results independently confirm the conclusions of B10, without using any model. Indeed, B10 were unable to fit the visibilities at higher spatial frequencies without introducing some continuous emission in the space between the rim and the star. They called this region the inner disk. We do not know whether the shape of this central source in the $K$-band image in the form of two spots is real or not, since in the simulated image this central source also seems to be decomposed into 3 single sources, which were not present in the model. It might be only the effect of the $(u, v)$ plane coverage, but we cannot exclude too that it might be due to a hot spot in the disk, although we should in principle then see it in the two images. The reconstructed images cannot help us to determine the origin of the inner disk emission, and ascertain whether it comes from hot gas or very refractory grains. These unknowns may be solved by combining high resolution spectroscopic observations in the near-infrared with advanced models that selfconsistently compute the emission of both dust and gas.

Finally, it remains unclear whether the non-zero value of the closure phases found by B10 is caused by the contribution from the inner disk not being exactly centered with the external ring. This might be the case when the disk surface is flaring and the system is seen with non-zero inclination. If the curvature of the surface of the disk probed by our images is large enough, then the ellipses tracing equal distances to the star will be shifted in the polar direction.

\subsection{Consequences on the image reconstruction}

Since image reconstruction in optical interferometry remains in its infancy, only a too sparse $(u, v)$ coverage is available to reconstruct an unambiguous image of a complex object and analyze it 


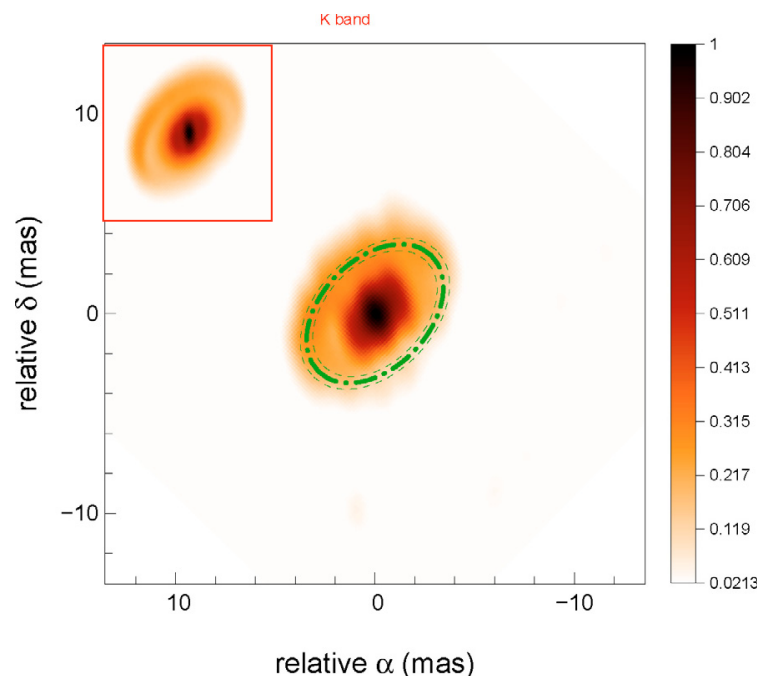

Fig. 6. Reconstructed image of the $\mathrm{B} 10$ model in the $K$ band, using a synthetic $(u, v)$ plane obtained with 3 quadruplets (A0-K0-G0-I1, D0H0-G1-I1, E0-G0-H0-I1) at VLTI and the CHARA baselines (S1-W1, W1-W2, S2-W2, E1-W1, E2-S2, S2-W1).

without the help of the model-fitting technique. Repeated comparisons between the model and the reconstructed image have to be performed to avoid over-interpreting the structures in the images. However, in all these cases, the image reconstruction technique remains the only technique able to perform modelindependent analysis of the data and is a powerful tool to give more credits to the models, derive tighter constraints, or even reveal unexpected structures.

In the future, two aspects of the image reconstruction will be important:

1. The homogeneity of the $(u, v)$ plane: thanks to several tests on the B10 model, we found that the global blobby aspect of the reconstructed image is almost certainly caused by the non-homogeneity of the $(u, v)$ plane. Indeed the reconstructed images from a homogeneous $(u, v)$ plane are smoother. The holes in the $(u, v)$ plane correspond to blobby or point-like structures in the reconstructed images, and the baselines for the observations have to be carefully chosen to map the $(u, v)$ plane as homogeneously as possible.

2. The quality of the measurements: the number of data points and their associated errors can clearly determine the dynamic range of the image. A larger number of data points and smaller error bars are needed to improve the dynamics of the image.

These aspects are illustrated in Fig. 6 where data on the B10 model are simulated with a $(u, v)$ plane for 3 quadruplets of telescopes at VLTI, as offered in the coming period P86 for the AMBER instrument and later for the PIONIER visitor instrument. The reconstructed image from these data contains fewer artifacts than those from the actual data set. The advent of the next generation imaging instruments at the VLTI, such as GRAVITY, MATISSE, and VSI, with up to 8 telescopes, will allow us to study in detail the inner disk structure, to detect planetary gaps and map outflows - bringing unambiguous constraints on crucial mechanisms for star and planet formation.

\section{Conclusion}

Since the renewal of optical interferometry in the mid-70's, imaging using aperture synthesis between different telescopes
Table A.1. Log of the data used for the image reconstruction.

\begin{tabular}{lllllll}
\hline \hline Interferom. & Obs. & $B_{P}(\mathrm{~m})$ & $\#$ meas. & Band Disp. & Refs. \\
\hline KI & 2003 & $72-78$ & 4 & $K$ & BB & M05 \\
IOTA & $2003-04$ & $10-28$ & 9 & $H$ & BB & M06 \\
CHARA & $2004-07$ & $86-313$ & 16 & $K$ & BB & T08 \\
VLTI/ & \multirow{2}{*}{2008} & \multirow{2}{*}{$13-128$} & 947 & $K$ & \multirow{2}{*}{35} & B10 \\
AMBER & & & 544 & $H$ & & \\
\hline
\end{tabular}

Notes. $B_{P}$ is the projected baselines; Disp. is the spectral dispersion. References are M05 (Monnier et al. 2005), M06 (Monnier et al. 2006), T08 (Tannirkulam et al. 2008), B10 (Benisty et al. 2010).

has been attempted for all possible astrophysical topics following the path opened by radio-interferometry with the Very Large Array. Unfortunately, optical interferometry requires precisions in the optical path delay $10^{3}$ to $10^{5}$ smaller than in the radio domain and therefore the development of large optical arrays has been slower. Although images of stellar surfaces or binaries have been obtained, no synthetic images have so far been possible at the milli-arcsecond scale of complex young stellar objects.

This paper is the first one attempting to reconstruct images of the close environment of a young star independent of any a priori model by gathering many interferometric measurements on the young star HD 163296 with various interferometers (VLTI, IOTA, KeckI, and CHARA). Using the Multi-Aperture Image Reconstruction Algorithm (MiRA), we have reconstructed images of HD 163296 in the $H$ and $K$ bands. The quality of the images compares well to those obtained 40 years ago with the first three antennas of the Very Large Array. To assess the reality of the features that are present, we have compared these images with images obtained from simulated data using the best known physical model of the environment of HD 163296 and reconstructed with the same conditions of $(u, v)$ plane and noise.

Those model-independent $H$ and $K$-band images of the surrounding of HD 163296 reveal several significant features that we can relate to the presence of an inclined asymmetric disk around HD 163296. We also detect a pre-eminent intensity at the location of dust sublimation above a structure extending from the central source with some differential effect along the azimuth that is not necessarily related to a puffed-up inner rim. Together with a slight offset of the central source compared to this bright ring, we proposed this to be the signature of the flared surface of a disk with a discontinuity caused by a opacity change.

These images confirm that the morphology of the close environment of young stars is more complex than the simple models used in the literature. We have also shown that having a more uniform $(u, v)$ plane coverage and higher measurement accuracies should help us to obtain higher quality images in the future.

Acknowledgements. The authors are grateful to the different institutions that trusted them in giving them guaranteed observing time with the VLTI to perform the first image of a complex young stellar object: CNRS through its national programs (ASHRA and PNPS), INAF and the AMBER consortium. This research has made use of NASA's Astrophysics Data System service, of the Jean-Marie Mariotti Center (JMMC) resources of the SIMBAD database, operated at CDS, Strasbourg, France, and of the Yorick, a free data processing language written by D. Munro (http: //yorick . sourceforge . net). M.B. acknowledges fundings from INAF (grant ASI-INAF I/016/07/0).

\section{Appendix A: The data set in detail}

The data used in this article comes from several interferometers. The name of these facilities, the observation date, the projected baselines, the number of measurements, the spectral dispersion, and the references have been summarized in Table A.1. 

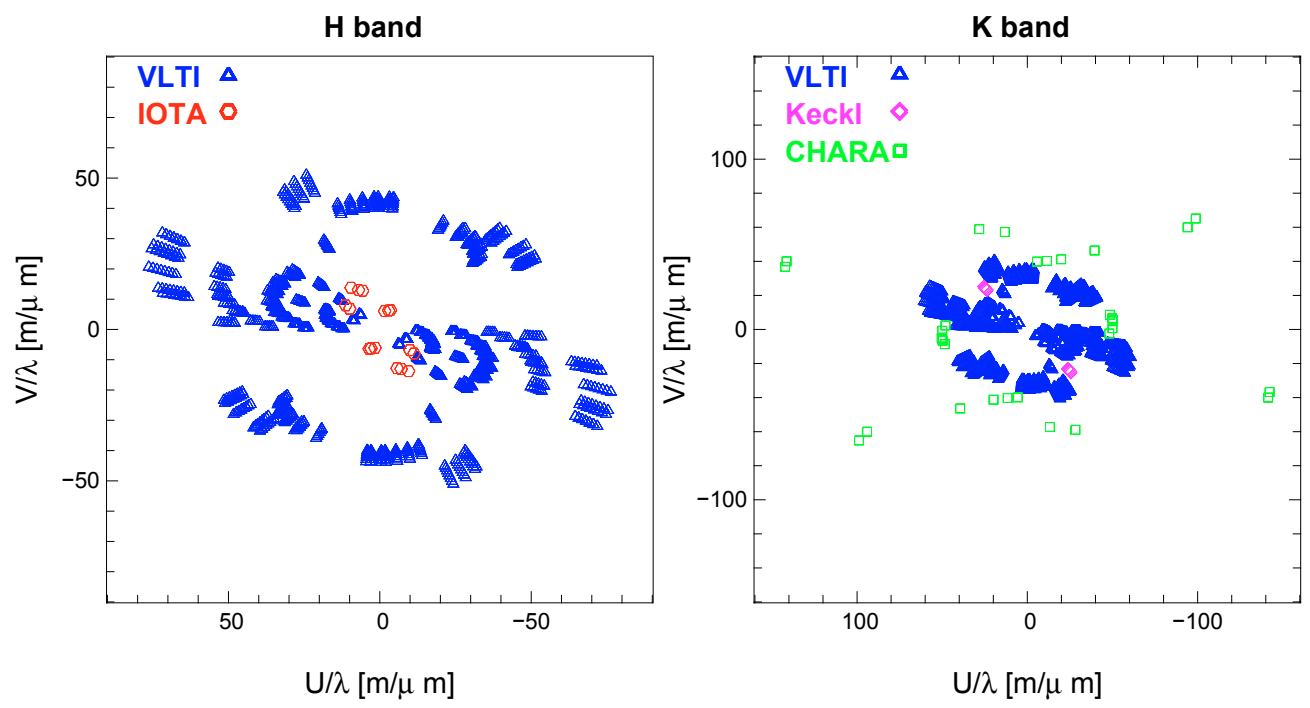

Fig. A.1. $(u, v)$ plane coverage of the data used for the image reconstruction in spatial frequencies for the $H(l e f t)$ and $K(r i g h t)$ bands. The different interferometers are plotted in different colors and symbols.
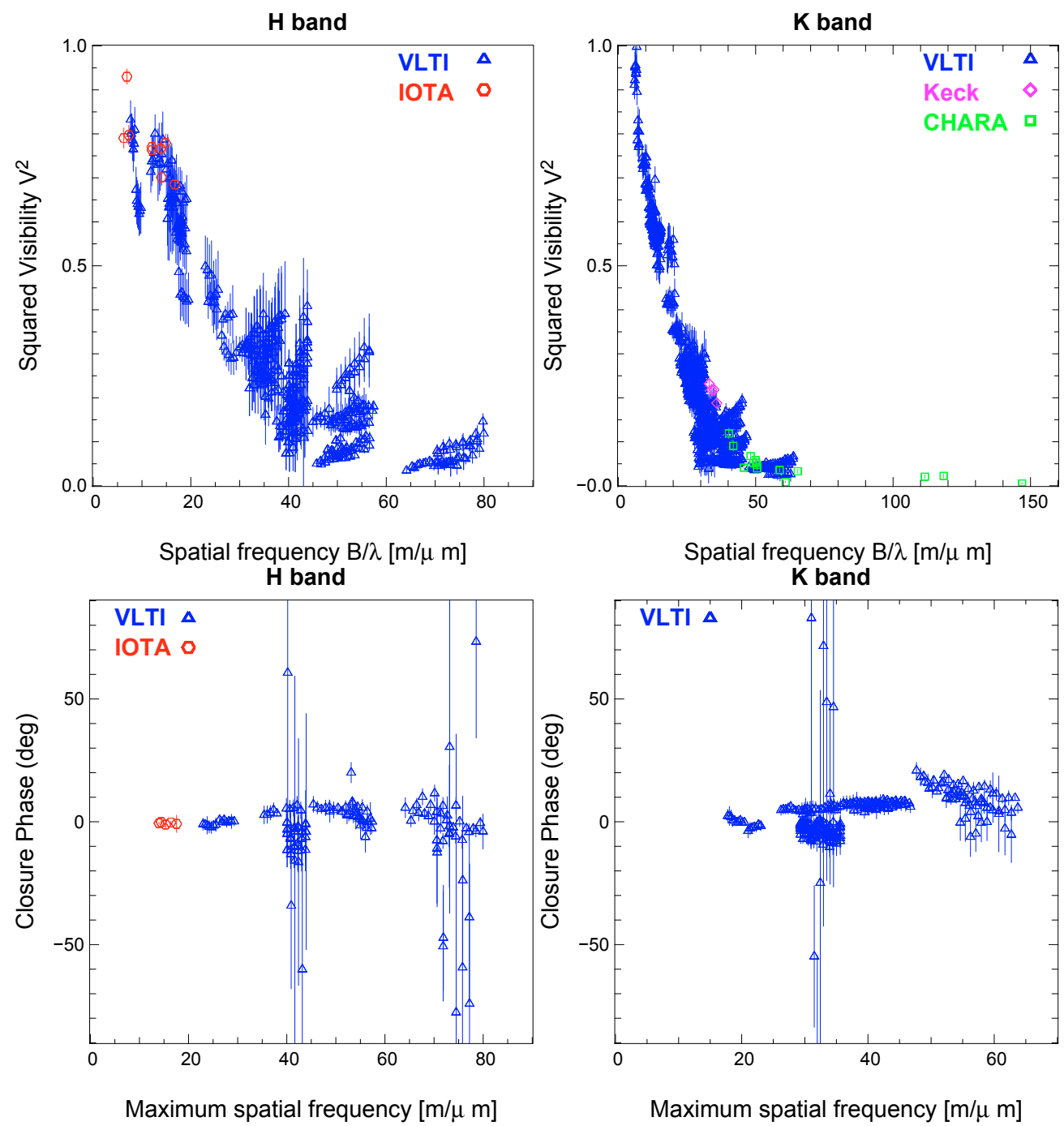

Fig. A.2. Squared visibilities (up) and closure phases (bottom) in the $H$ (left) and $K$ (right) bands. The different interferometers are plotted in different colors and symbols.

The $(u, v)$ plane in the $H$ and $K$ bands is plotted in Fig. A.1. The data (squared visibilities and closure phases) is displayed in Fig. A.2. For a better view, a zoom on the VLTI data is shown in Fig. A.3, with various colors for different ranges of baseline position angles. 

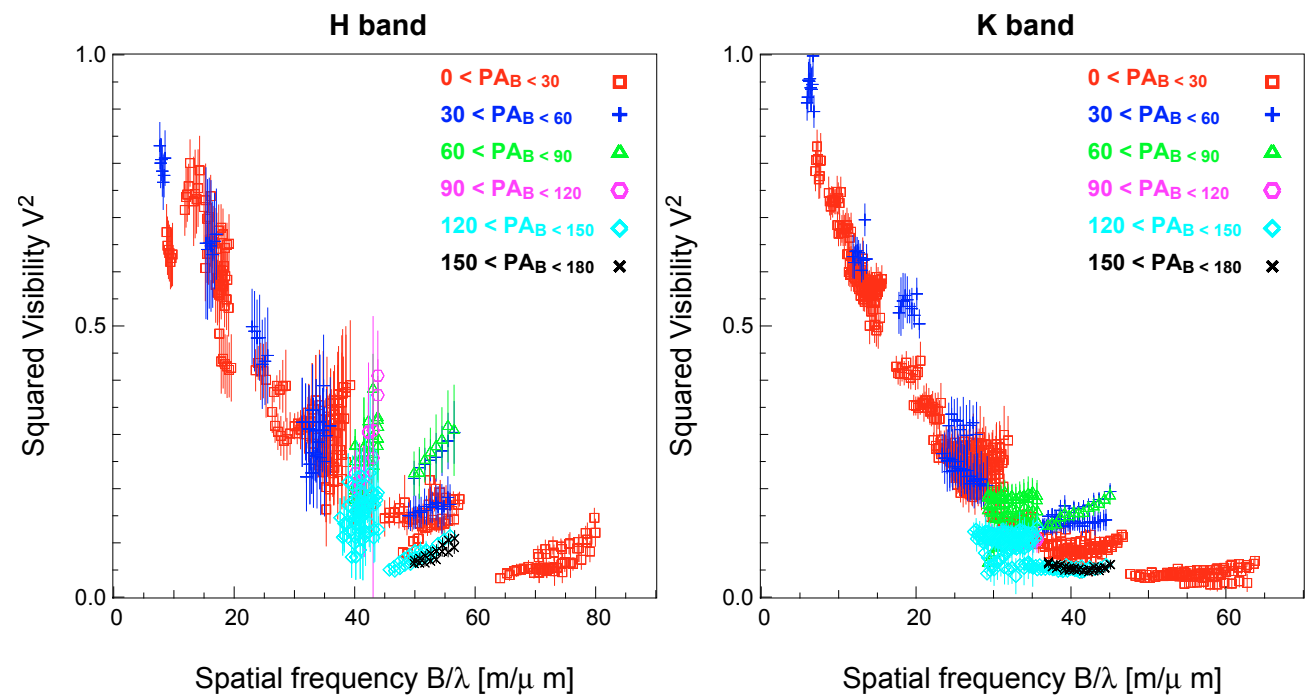

Fig. A.3. Squared visibilities in the $H$ (left) and $K$ (right) bands for the VLTI/AMBER data. The different colors and symbols correspond to different baseline position angle ranges.
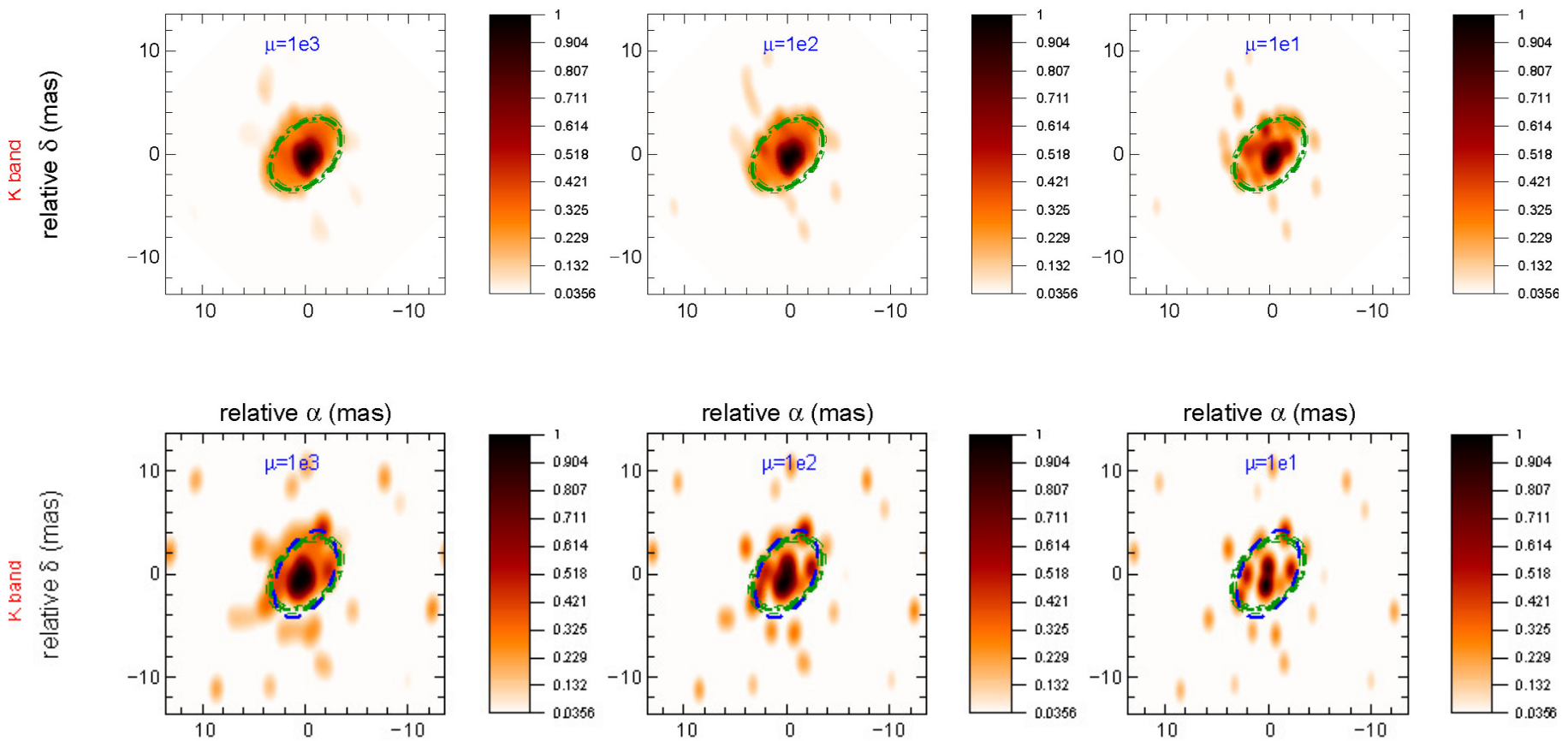

relative $\alpha$ (mas)

relative $\alpha$ (mas)

relative $\alpha$ (mas)

Fig. B.1. Reconstructed images of HD 163296 in the $K$ band from simulated data of the B10 model (up) and from the real data (bottom), for 3 different values of the weight factor $\mu$. Conventions as in Fig. 1 .

\section{Appendix B: The effect of the regularization weight}

The systematic tests performed in RTM10 shows that a weight factor $\mu$ can be associated with a regularization term to within an order of magnitude. In this appendix, we illustrate the effect of the $\mu$ factor on the reconstructed images, therefore determining the influence of $\mu$ and demonstrating that $\mu=10^{2}$ is the best value.

The upper part of Fig. B.1 presents the reconstructed images from the B10 model in the $K$ band for 3 different values of the weight factor $\mu(1000,100,10)$. When comparing them to the model (see the upper left corner of Fig. 3), the following information can be extracted:

- In the left part of Fig. B.1, the image is too regularized, meaning that too much weight is put on the regularization term and not enough on the data. The reconstructed image is too smooth and several structure are not visible, because of the lack of distinction between the external ring and the internal disk.

- In the right part of Fig. B.1, the image is insufficiently regularized, meaning that too much weight has been placed on the data. The reconstructed image is far more blobby than the original and the flux in the internal disk starts to disappear. 
- The ideal weight factor is shown in the middle part of Fig. B.1. In this figure, all the different characteristics of the model are presented and it seems to provide the best reconstruction between the three.

The same analysis can be performed on the reconstructed images from the real data and are illustrated in the bottom part of Fig. B.1.

\section{References}

Balbus, S. A., \& Hawley, J. F. 1991, ApJ, 376, 214

Baldwin, J. E., \& Haniff, C. A. 2002, Phil. Trans. R. Soc. London, Ser. A, 360, 969

Benisty, M., Natta, A., Isella, A., et al. 2010, A\&A, 511, A74 (B10)

Boss, A. P. 1997, Science, 276, 1836

Cotton, W., Monnier, J., Baron, F., et al. 2008, in SPIE Conf. Ser., 7013

Deleuil, M., Bouret, J., Catala, C., et al. 2005, A\&A, 429, 247

Devine, D., Grady, C. A., Kimble, R. A., et al. 2000, ApJ, 542, L115

Dullemond, C. P., Dominik, C., \& Natta, A. 2001, ApJ, 560, 957

Grady, C. A., Devine, D., Woodgate, B., et al. 2000, ApJ, 544, 895

Haubois, X., Perrin, G., Lacour, S., et al. 2009, A\&A, 508, 923

Henning, T., \& Meeus, G. 2009, in Physical Processes in Circumstellar Disks around Young Stars, ed. P. J. V. Garcia, Theoretical Astrophysics Series (Chicago: University Press), in press [arXiv: 0911.1010]

Hogg, D. E., MacDonald, G. H., Conway, R. G., \& Wade, C. M. 1969, AJ, 74, 1206

Isella, A., Testi, L., Natta, A., et al. 2007, A\&A, 469, 213

Kraus, S., Weigelt, G., Balega, Y. Y., et al. 2009, A\&A, 497, 195
Lacour, S., Meimon, S., Thiébaut, E., et al. 2008, A\&A, 485, 561

Lacour, S., Thiéaut, E., Perrin, G., et al. 2009, ApJ, 707, 632

Lawson, P. R., 2000, Principles of Long Baseline Stellar Interferometry

Le Bouquin, J., Lacour, S., Renard, S., et al. 2009, A\&A, 496, L1

Malbet, F., \& Perrin, G. 2007, New Astron. Rev., 51, 563

Mannings, V., \& Sargent, A. I. 1997, ApJ, 490, 792

Mayer, L., Quinn, T., Wadsley, J., \& Stadel, J. 2002, Science, 298, 1756

Millan-Gabet, R., Malbet, F., Akeson, R., et al. 2007, Protostars and Planets V, 539

Monnier, J. D. 2003, Rep. Progr. Phys., 66, 789

Monnier, J. D., Millan-Gabet, R., Billmeier, R., et al. 2005, ApJ, 624, 832

Monnier, J. D., Berger, J., Millan-Gabet, R., et al. 2006, ApJ, 647, 444

Monnier, J. D., Zhao, M., Pedretti, E., et al. 2007, Science, 317, 342

Montesinos, B., Eiroa, C., Mora, A., \& Merín, B. 2009, A\&A, 495, 901

Natta, A., Testi, L., Neri, R., Shepherd, D. S., \& Wilner, D. J. 2004, A\&A, 416, 179

Strong, D., \& Chan, T. 2003, Inverse Problems, 19, S165

Swartz, D. A., Drake, J. J., Elsner, R. F., et al. 2005, ApJ, 628, 811

Tannirkulam, A., Monnier, J. D., Millan-Gabet, R., et al. 2008, ApJ, 677, L51

Thiébaut, E. 2002, in SPIE Conf. Ser. 4847, ed. J.-L. Starck, \& F. D. Murtagh, 174

Thiébaut, E. 2005, in NATO ASIB Proc. 198: Optics in astrophysics, ed. R. Foy, \& F. C. Foy, 397

Thiébaut, E. 2008, in SPIE Conf. Ser. 7013

Thiébaut, É., \& Giovannelli, J.-F. 2009, 2010, ISPM, 27, 970

van den Ancker, M. E., de Winter, D., \& Tjin A Djie, H. R. E. 1998, A\&A, 330, 145

Wassell, E. J., Grady, C. A., Woodgate, B., Kimble, R. A., \& Bruhweiler, F. C. 2006, ApJ, 650, 985

Zhao, M., Gies, D., Monnier, J. D., et al. 2008, ApJ, 684, L95

Zhao, M., Monnier, J. D., Pedretti, E., et al. 2009, ApJ, 701, 209 